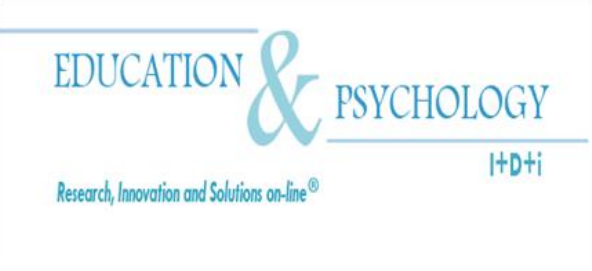

\title{
Desarrollo de la competencia emocional de maestros y alumnos en contextos escolares
}

\section{Pérez-Escoda, N. ${ }^{1}$, Filella, G. ${ }^{2}$, Alegre, A. ${ }^{3}$ y Bisquerra, R. ${ }^{1}$}

1 Departamento de Métodos de Investigación y Diagnóstico en Educación de la Universidad de Barcelona, Barcelona, España

2 Departamento de Pedagogía y Psicología de la Universidad de Lleida, Lleida, España

3 Departamento de Educación Infantil y Primaria de la Universidad de East Stroudsburg, East Stroudsburg, Pennsylvania, EE.UU.

\section{España / EEUU}




\section{Resumen}

Introducción. En este trabajo se presenta la evaluación de dos programas de formación, uno dirigido a maestros y otro a alumnos con el objetivo de mejorar el bienestar personal y social mediante el desarrollo de la competencia emocional (conciencia emocional, regulación emocional, autonomía emocional, competencia social y competencias para la vida).

Método. Han participado en la experiencia 92 maestros y 423 niños de 6 a 12 años de diversos centros de educación primaria. Se ha utilizado un diseño cuasiexperimental pretestpostest con grupo control para evaluar la eficacia de cada uno de los programas de formación. Para ello se han aplicado diferentes instrumentos para medir la evolución la competencia emocional y sus cinco dimensiones.

Resultados. La investigación demuestra una mejora significativa de la competencia emocional de los participantes al final de la intervención, junto a un mejor clima de relación en las escuelas.

Discusión y Conclusión. Se corrobora la opinión de los expertos en el tema quienes afirman que las competencias emocionales tales como: la conciencia emocional, la regulación emocional, la autonomía emocional, las competencias sociales y las habilidades de vida y bienestar pueden enseñarse y aprenderse.

Palabras Clave: Educación Emocional, inteligencia emocional, aprendizaje social y emocional, evaluación de programas, escuela primaria, evaluación de la competencia emocional. 


\title{
Developing the emotional competence of teachers and pupils in school contexts
}

\begin{abstract}
Introduction. This paper describes the evaluation of two training programmes, one targeted at teachers and the other at pupils, the aim of both being to improve personal and social wellbeing through the development of emotional competence (emotional awareness, emotional regulation, emotional autonomy, social competence and life competencies).

Method. Participants were 92 teachers and 423 children aged 6-12 years from various primary schools. A quasi-experimental pre-test/post-test design with control group was used to evaluate the efficacy of the two training programmes, with different instruments being applied to measure the evolution of emotional competence and its five dimensions.

Results. The results showed a significant improvement in the emotional competence of participants at the end of the intervention, together with a better relational climate in schools.

Discussion and Conclusion. They also corroborate current expert opinion that emotional competencies such as emotional awareness, emotion regulation, emotional autonomy, social competence, and life and well being competencies can be taught and learnt.
\end{abstract}

Keywords: Emotional education, emotional intelligence, social and emotional learning, programme evaluation, primary school, assessment of emotional competence.

Received: 07/25/12 Initial acceptance: 08/30/12 Final acceptance: 11/08/12 


\section{Introducción}

Existen buenas razones para considerar la educación emocional como parte del espíritu de la época que caracteriza el inicio del siglo XXI. Fernández Berrocal y Aranda Ruiz (2008) consideran que hoy en día es completamente necesario educar la inteligencia emocional de los niños. La educación emocional cuenta con fuertes fundamentos teóricos que son objeto de constante revisión y actualización (Bechara, Tranel, y Damasio, 2000; Goleman, 1995; Mayer, Caruso y Salovey, 2001; Bisquerra, 2000, 2009; Gardner, 1993; Cherniss, 2000; Hedlund y Sternberg, 2000, entre muchos otros). Expertos en la materia coinciden en que existen ciertas competencias emocionales que se pueden aprender, y que existe la necesidad de integrarlas en la educación temprana y continua de todo el mundo (Mayer, Caruso y Salovey, 2002; Salovey et al, 2000; Collaborative for Academic, Social and Emotional Learning, 2003; Petrides, Frederickson y Furnham, 2004; Saarni, 2000; Sroufe, 2000). Estas competencias pueden resultar de enorme utilidad cuando se enfrenta a una amplia variedad de situaciones cotidianas (Humphrey, 2010; Saarni, 2000).

Algunas revisiones han investigado los resultados de varios programas de educación emocional (Catalano et al, 2002; Greenberg, Weissenberg, O'Brien, Zins, Fredericks, Resnik y Elias, 2003; Clouder et al, 2008;. Di Fabio et al, 2011), lo que demuestra sus efectos positivos sobre los niños en una variedad de contextos (desde la educación infantil hasta la escuela secundaria, y en las zonas urbanas, suburbanas y rurales). En concreto, el metaanálisis realizado por Greenberg et al. (2003), que analizó 300 programas de aprendizaje social y emocional, demostró que este tipo de programas conducen a mejoras significativas en el rendimiento escolar de los niños. Además, Nelis et al (2009) encontró que los adultos jóvenes que participaron en un curso intensivo de formación emocional habían mejorado sus niveles de inteligencia emocional. Esta mejoría se mantuvo seis meses después.

En su estudio, Nelis et al (2009) concluyeron que la gran mayoría de los programas de formación en inteligencia emocional sufren de tres tipos de problemas de diseño: 1) la mayoría no se basan en un modelo teórico sólido, 2) que actúan sólo en ciertas dimensiones de la inteligencia emocional, y 3) que no utilizan los grupos control.

En vista de lo anterior, existe una necesidad de un estudio de investigación el cual se base en el programa de educación emocional siendo investigado desde un modelo teórico sólido, se dirija a un grupo global de las competencias emocionales, y utilice un diseño 
experimental con grupo control. El presente estudio tiene por objeto investigar la eficacia de un programa de educación emocional que se incluye estas tres cuestiones de diseño: se basa en un modelo teórico sólido (Bisquerra y Pérez, 2007), se dirige a un grupo amplio de competencias emocionales, y utiliza un grupo experimental y un grupo control para encontrar evidencia de efectos significativos.

El modelo teórico de competencia emocional de Bisquerra y Pérez (2007) propone que las competencias emocionales se pueden agrupar en cinco grandes dimensiones: Conciencia emocional, regulación emocional, autonomía personal, la competencia social y competencias para la vida y el bienestar. La conciencia emocional integra la capacidad de ser consciente de las propias emociones, y la capacidad de captar el clima emocional en un contexto específico. Regulación emocional se refiere a la capacidad de utilizar las emociones adecuadamente. Se requiere ser consciente de la relación entre emoción, cognición y comportamiento, y tener estrategias eficaces de afrontamiento y la capacidad de auto-generar emociones positivas. Autonomía personal incluye un conjunto de características relacionadas con el auto-manejo de emociones tales como: la autoestima, la actitud positiva ante la vida, la responsabilidad, la capacidad de analizar críticamente las normas sociales, la capacidad para buscar ayuda y recursos cuando sea necesario, y las creencias de autoeficacia. La competencia social se refiere a la capacidad de establecer relaciones positivas con otras personas. Se requiere el dominio de habilidades sociales básicas, la comunicación efectiva, el respeto por los demás, la conducta prosocial asertiva. Competencias para la vida y el bienestar se refiere a la capacidad de mostrar un comportamiento responsable y adecuado para resolver los problemas de tipo personal, familiar, profesional, sociales dirigidos a la mejora de la persona y de la sociedad del bienestar.

El modelo de Bisquerra y Pérez se puede considerar un modelo de IE porque incorpora tanto rasgos cognitivos como dimensiones de la personalidad. En comparación con los modelos típicos capacidad de inteligencia emocional (por ejemplo, Mayer y Salovey, 2007), tiene la ventaja de ser más amplio. En comparación con otros modelos de la IE (Petrides, Frederick hijo y Furnham, 2004), tiene la ventaja de estar más jerárquicamente estructurado facilitando el posterior desarrollo de programas de intervención (Bisquerra y Pérez, 2007). 
El programa de intervención desarrollado, basado en el modelo de competencia emocional de Bisquerra y Pérez (2007), ofrece secciones de formación específicos para cada una de las cinco competencias emocionales incluidas en el modelo. Para la conciencia emocional, el programa ayuda a los participantes a ampliar su vocabulario emocional y aprender a reconocer sus propias emociones. Para capacitar en la regulación emocional, los participantes aprenden técnicas de relajación. Para la autonomía personal, aprenden a reconocer sus propias cualidades y utilizarlas para aumentar su autonomía. Para desarrollar la competencia social, los participantes aprenden a reconocer las emociones de los demás y de expresar sus propias emociones en formas socialmente apropiadas. También aprenden técnicas de resolución de conflictos, la asertividad y la empatía. En cuanto a las competencias para la vida y el bienestar, los participantes aprenden a ser críticamente reflexivos, para mantener un equilibrio entre sus intereses personales y las necesidades del medio ambiente. El programa de entrenamiento utiliza diferentes técnicas de desarrollo de habilidades para integrar la teoría de una manera vivencial y participativa, a través de dinámicas de grupo, debates, reflexiones y ejercicios de juego de roles.

Por lo tanto, el objetivo del estudio es investigar la eficacia de un programa de educación emocional que se basa en un modelo teórico sólido, se dirige a un grupo amplio de competencias emocionales, y utiliza un grupo de control como medio para evaluar los resultados. El programa se divide en dos subprogramas. El primer subprograma fue impartido por los investigadores y dirigido a la educación emocional de los maestros en varias escuelas de dos ciudades españolas. El segundo subprograma fue impartido por los maestros capacitados y orientados hacia sus estudiantes. Como consecuencia de ello, se llevaron a cabo dos estudios: uno para evaluar la eficacia del programa de educación emocional para maestros (Estudio 1), y el otro para evaluar la eficacia del programa equivalente que los maestros aplican a sus alumnos (Estudio 2).

\section{Estudio 1}

En este estudio se analizó el efecto del programa de educación emocional para maestros en lo relativo al desarrollo de la competencia emocional de los participantes. Investigaciones anteriores han mostrado que los maestros comprenden la necesidad de mejorar sus propias competencias sociales y emocionales, como requisito previo a reforzar y mejorar las habilidades y competencias de sus estudiantes (Triliva y Poulou, 2006). Por lo 
tanto, nuestro programa de intervención se inició con la formación de los maestros. En consonancia con el modelo de Bisquerra y Pérez (2007) la atención se centró tanto en la competencia emocional en general como en cada una de sus cinco dimensiones: la conciencia emocional, regulación emocional, autonomía emocional, competencia social, y competencias para la vida y el bienestar.

El programa consta de 30 horas de formación, y se llevó a cabo en sesiones de una hora a la semana, durante 30 semanas, en un período de nueve meses. Las sesiones de capacitación se llevaron a cabo en las escuelas donde trabajaban los maestros. Se asignaron seis horas de formación a cada competencia emocional descrita anteriormente. Los maestros fueron formados por los autores de los mismos estudios.

Para determinar la eficacia del programa, fueron necesarias tres tipos de pruebas. En primer lugar, era importante saber si el programa había tenido algún efecto en la competencia emocional de los participantes. En segundo lugar, saber si el programa había sido efectivo, dando lugar a resultados positivos para los participantes. En concreto, cualquier mejora en la competencia emocional de los maestros que se reflejara en su capacidad para afrontar mejor los retos del empleo y experimentar menos estrés. En este sentido, varios estudios (Brackett et al, 2010; Extremera, Fernández-Berrocal y Durán, 2003) han demostrado que cuando los maestros gozan de altos niveles de inteligencia emocional experimentan un menor número de pensamientos negativos y menos agotamiento. Por último, una escuela donde los maestros hubieran mejorado su capacidad emocional, y la reducción de sus niveles de estrés debería experimentar un ambiente escolar más armonioso.

\section{Hipótesis del Estudio 1}

Esperábamos que, en comparación con el grupo control, los maestros participantes en el programa de educación emocional experimentaría el siguiente: 1) una mejora de su competencia emocional total y en cada una de sus dimensiones: la conciencia emocional, regulación emocional, autonomía emocional, competencia social y competencias para la vida y el bienestar, 2) la reducción de los niveles de estrés, y 3) una mejora en el clima institucional.

\section{Método del Estudio 1}




\section{Participantes}

La muestra estaba compuesta por 92 maestros, de los cuales se asignó el 62\% al grupo experimental (GE). Los participantes fueron seleccionados a través de un muestreo intencional de subgrupos homogéneos. Los maestros en el GE pertenecían a tres escuelas estatales de Educación Primaria, mientras que el grupo control (GC) ejercía en otras cinco escuelas de este tipo. Estas escuelas se encuentran en barrios de un nivel medio-bajo. Todos los maestros tenían por lo menos un título de licenciatura, y su edad media era de 42 años. El $88 \%$ de los participantes eran mujeres, lo que refleja el perfil típico de la maestra de escuela de primaria en España.

\section{Procedimiento}

Se utilizó un diseño pre-test/post-test cuasi-experimental con grupo control para evaluar la eficacia del programa de formación. Todos los instrumentos fueron administrados a los maestros de ambos grupos. El intervalo entre el pre-test y post-test de evaluación fue de nueve meses.

\section{Instrumentos}

Competencia Emocional. La competencia emocional se mesuró con el Cuestionario de Desarrollo Emocional para Adultos (CDE-A), desarrollado por el Grupo de Investigación en Orientación Psicopedagógica (GROP) de la Universidad de Barcelona. Se trata de un autoinforme basado en el modelo teórico de la educación emocional desarrollado por Bisquerra y Pérez (2007). Consta de 48 ítems (por ejemplo, "me resulta difícil hablar con mis amigos sobre mis sentimientos"), cada uno de los cuales se califica en una escala de 11 puntos de Likert de 0 a 10, y proporciona una puntuación global y otra para cada una de las cinco dimensiones del modelo. La escala fue validada por un panel de 11 expertos que combina cada elemento a la subescala correspondiente de acuerdo con la meta de la escala y el modelo teórico. Todos los artículos obtenidos anteriormente el $80 \%$ de acuerdo entre todos los expertos para cada escala (Pérez-Escoda, et al, 2010). Los autores presentaron la escala a un análisis factorial exploratorio con rotación Varimax. El análisis de los valores propios (screeplot) reveló cinco factores que se denominan conciencia emocional, regulación emocional, 
autonomía emocional, competencia social, y competencias para la vida y el bienestar, como lo confirma el modelo teórico. Los cinco factores explican el 50\% de la varianza. Los autores informan medidas de fiabilidad (alfa de Cronbach) de 0.92 para la escala completa y superior a 0.69 para cada una de las cinco dimensiones del modelo. En el presente estudio los valores del alfa de Cronbach fueron 0.91 para la escala total, 0.67 para la conciencia emocional, 0.79 para la regulación emocional, 0.61 para la autonomía emocional, 0.68 para la competencia social y 0.72 para las competencias de vida y el bienestar.

Nivel de estrés. Para medir el nivel de tensión de los participantes se elaboró una escala de cinco ítems. Como la percepción del estrés depende de múltiples factores, esta escala se basó en las dimensiones propuestas por Novak (1991, 2008): personal, interpersonal y de contexto (situación de la salud, el empleo y la familia). Los participantes puntuaban de 0 (muy mal) a 10 (excelente) en función de lo satisfechos que estaban con cada dimensión. La consistencia interna de la escala (medida por el alfa de Cronbach) fue de 0.57.

Clima institucional. Este aspecto se centró específicamente en el clima relacional en la escuela: las relaciones entre los maestros, entre los alumnos y con las familias de los alumnos. Los participantes puntuaban de 0 (muy mal) a 10 (excelente) cómo de bueno valoraban el clima relacional en cada nivel de la escuela. Se calculó el coeficiente de clima institucional. La escala utilizada para evaluar este aspecto tenía una consistencia interna (medida por el alfa de Cronbach) de 0.63.

\section{Análisis de los datos}

Debido a la distribución normal de los errores y de la relación lineal entre las variables dependientes e independientes, los datos de la competencia emocional, el clima institucional y los niveles de estrés se sometieron a un análisis de modelo lineal general. Para cada competencia, fue aplicado el modelo lineal general para medidas repetidas. Las variables intrasubjects fueron las fases, y la variable intersubjects el grupo. Debido a que los factores eran sólo de dos categorías, no se realizaron análisis ad hoc de contraste. La función de enlace se utiliza como función de la identidad, las variables independientes se consideraron factores y la variable dependiente se tomó como continua. Este análisis se prefirió a otros tipos de análisis globales porque nos permitió respetar los niveles de significancia establecidos "a priori". 


\section{Resultados del Estudio 1}

\section{Descriptivos}

La Tabla 1 muestra los estadísticos descriptivos de cada factor del CDE-A, y variables cada uno.

Tabla 1. Estadísticos descriptivos

\begin{tabular}{|c|c|c|c|c|c|}
\hline & & \multicolumn{2}{|c|}{ Pre-test } & \multicolumn{2}{|c|}{ Post-test } \\
\hline \multirow{9}{*}{ GE } & & Mean & SD & Mean & SD \\
\hline & Conciencia emocional & 7.05 & $(1.06)$ & 7.27 & $(1.19)$ \\
\hline & Regulación emocional & 5.28 & $(1.10)$ & 5.86 & $(1.36)$ \\
\hline & Autonomía emocional & 6.05 & $(1.02)$ & 6.44 & $(1.14)$ \\
\hline & Competencia social & 5.68 & $(1.01)$ & 5.87 & $(1.37)$ \\
\hline & Competencias de vida y bienestar & 7.04 & $(.97)$ & 7.35 & $(1.20)$ \\
\hline & Total & 6.12 & $(.81)$ & 6.49 & $(1.10)$ \\
\hline & Niveles de estrés & 16.68 & $(4.74)$ & 14.39 & $(3.55)$ \\
\hline & Clima institucional & 25.00 & $(4.75)$ & 27.73 & $(3.56)$ \\
\hline \multirow{8}{*}{ GC } & Conciencia emocional & 7.28 & $(1.17)$ & 7.15 & $(1.24)$ \\
\hline & Regulación emocional & 5.73 & $(1.44)$ & 5.69 & $(1.38)$ \\
\hline & Autonomía emocional & 6.08 & $(1.22)$ & 6.09 & $(1.28)$ \\
\hline & Competencia social & 5.59 & $(1.37)$ & 5.70 & $(1.38)$ \\
\hline & Competencias de vida y bienestar & 6.89 & $(1.45)$ & 6.95 & $(1.48)$ \\
\hline & Total & 6.24 & $(1.14)$ & 6.24 & $(1.19)$ \\
\hline & Niveles de estrés & 16.51 & $(4.88)$ & 16.61 & $(4.94)$ \\
\hline & Clima institucional & 25.54 & $(3.93)$ & 25.87 & $(3.81)$ \\
\hline
\end{tabular}

\section{Resultados del programa}

La aplicación del modelo lineal general con 1,11 grados de libertad para las variables competencias emocionales, y 1,75 grados de libertad para los niveles de estrés y las variables 
del clima institucional se puede ver en la Tabla 2. Las diferencias entre las etapas pre-test y post-test, entre el GE y el GC.

Tabla 2. Resultados generales de modelo lineal general

\begin{tabular}{|c|c|c|c|}
\hline $\begin{array}{c}\text { Competencia } \\
\text { Emocional }\end{array}$ & Factor & $\mathrm{F}$ & $\mathrm{P}$ \\
\hline \multirow{3}{*}{$\begin{array}{l}\text { Conciencia } \\
\text { emocional }\end{array}$} & Fase (pre-post) & .016 & .9 \\
\hline & Grupo (GE-GC) & .002 & .961 \\
\hline & Fase*Grupo & 2.435 & .122 \\
\hline Regulación & Fase (pre-post) & 2.771 & .1 \\
\hline \multirow[t]{2}{*}{ emocional } & Grupo (GE-GC) & .603 & .439 \\
\hline & Fase*Grupo & 25.773 & .000 \\
\hline Autonomía & Fase (pre-post) & 1.970 & .163 \\
\hline \multirow[t]{2}{*}{ emocional } & Grupo (GE-GC) & .011 & .921 \\
\hline & Fase*Grupo & 2.369 & .131 \\
\hline Competencia & Fase (pre-post) & .107 & .750 \\
\hline \multirow[t]{2}{*}{ Social } & Grupo (GE-GC) & .540 & .464 \\
\hline & Fase*Grupo & 5.288 & .023 \\
\hline Competencias de & Fase (pre-post) & .539 & .464 \\
\hline \multirow[t]{2}{*}{ vida y bienestar } & Grupo (GE-GC) & .880 & .350 \\
\hline & Fase*Grupo & 3.595 & .061 \\
\hline Total Emotional & Fase (pre-post) & .196 & .659 \\
\hline \multirow[t]{2}{*}{ Competence } & Grupo (GE-GC) & .026 & .872 \\
\hline & Fase*Grupo & 10.074 & .000 \\
\hline \multirow[t]{3}{*}{ Niveles de estrés } & Fase (pre-post) & .078 & .781 \\
\hline & Grupo (GE-GC) & .128 & .721 \\
\hline & Fase*Grupo & 9.231 & .000 \\
\hline \multirow{3}{*}{$\begin{array}{l}\text { Clima } \\
\text { institucional }\end{array}$} & Fase (pre-post) & 9.879 & .000 \\
\hline & Grupo (GE-GC) & .146 & .703 \\
\hline & Fase*Grupo & 13.373 & .000 \\
\hline
\end{tabular}

No hubo diferencias significativas entre las etapas pre-test y post-test, ni entre el GE y el GC para cualquiera de las cinco competencias emocionales o del coeficiente de competencia emocional general. Sin embargo, hubo una interacción significativa entre grupo 
y fase* para la regulación emocional, competencia social y la competencia emocional global, con sólo el grupo de intervención (GE) que muestra un aumento significativo en las tres dimensiones. Además, hubo interacción significativa entre grupo y fase*, para el nivel de estrés y la percepción del clima institucional. En ambos casos, sólo el GE reportó mejoras significativas.

\section{Discusión del Estudio 1}

Los resultados presentados anteriormente muestran que el programa de educación emocional para maestros produce mejoras significativas en el desarrollo de ciertas competencias emocionales entre los participantes. Sin embargo, la intervención no ha desarrollado en la misma medida las cinco dimensiones de la competencia emocional. Las mejoras más notables experimentadas por los maestros eran las de la regulación emocional y competencia social. Estos resultados se han visto respaldados por el hecho de que los maestros del GC, que no participaron en el programa, no experimentaron cambios estadísticamente significativos en la competencia total o en cualquiera de las cinco dimensiones estudiadas. Se puede concluir, por lo tanto, que el programa de educación emocional era al menos parcialmente eficaz.

Además, los maestros que participaron en el programa también han experimentado una mejora en la percepción sobre el clima institucional, así como una reducción significativa del estrés percibido. Esto confirma que la intervención es un recurso eficaz que puede ayudar a optimizar las relaciones interpersonales en la escuela, tener un impacto positivo en la calidad de vida de las personas que trabajan allí.

\section{Estudio 2}

Este estudio analizó la efectividad del Programa de Educación Emocional para el desarrollo de la competencia emocional en niños de primaria. El programa fue administrado por los docentes que recibieron la capacitación en el Estudio 1. Los contenidos de este programa de capacitación fueron esencialmente los mismos que los utilizados en el programa de capacitación para los maestros. Cada maestro adaptó los contenidos y actividades a las necesidades de cada grupo específico de niños. La formación se ofreció en las escuelas y en el 
horario normal, dedicando una sesión de una hora una vez por semana durante veinte semanas.

\section{Hipótesis del Estudio 2}

Esperábamos que en comparación con el grupo control, los niños que participaran en el programa de educación emocional (el grupo experimental) experimentaría una mejora en su capacidad emocional total y en cada una de las cinco sub-competencias: conciencia emocional, regulación emocional, autonomía emocional, competencia social, y competencias de vida y bienestar.

\section{Método del Estudio 2}

\section{Participantes}

Los participantes fueron 423 alumnos de 6-12 años, el 51,3\% de los cuales eran varones. Todos los niños asistían a escuelas públicas en barrios de nivel medio-bajo. El grupo experimental (GE) incluyó 223 alumnos, y los 200 restantes constituyeron el grupo de control (GC). La participación de los alumnos dependía de la aplicación voluntaria de la formación que habían recibido los maestros en el estudio 1.

\section{Procedimiento}

El programa de educación emocional para niños se componía de un total de 20 actividades distribuidas en bloques temáticos correspondientes a las cinco dimensiones del modelo teórico. En el GE se aplicó semanalmente una actividad en el marco del plan de acción tutorial. Las sesiones fueron siempre dirigidas por el profesor-tutor, se fomentó la implicación de los alumnos a partir de un trabajo individual y grupal mediante sesiones participativas como estrategia clave para la adquisición de competencias emocionales. Los efectos del programa se estudiaron por medio de un diseño pre-test/post-test cuasiexperimental con un grupo control. El intervalo entre las evaluaciones pre-test y post-test fue de nueve meses.

\section{Instrumentos}


Con el fin de evaluar el desarrollo de las competencias propuestas por el programa, se utilizaron dos instrumentos diferentes adecuados a la edad de los alumnos.

Debido a la carencia de escalas disponibles para medir el desarrollo emocional de los niños de ciclo inicial de educación primaria (6-8 años), fue necesario elaborar un instrumento de observación ad hoc. Se basa en el modelo teórico mencionado de Bisquerra y Pérez (2007) y nos ha permitido estimar la competencia emocional general de los niños y cada una de las cinco dimensiones. El instrumento de observación utilizado consta de 79 ítems, de los cuales 25 se refieren a los comportamientos a través del cual los niños demuestran su conciencia emocional (por ejemplo, si identificar la tristeza en los demás), 8 se refieren a la regulación emocional (por ejemplo, si puede pedir ayuda a otros), 12 se refieren a la autonomía emocional (por ejemplo, si identifica sus limitaciones), 27 se refieren a la competencia social (por ejemplo, si puede defenderse a si mismo o sus derechos), y 7 se refieren a competencias para la vida y el bienestar (por ejemplo, si puede mantener una actitud positiva hacia los cambios). La evaluación de la competencia emocional de cada niño estaba basada en las percepciones de su profesor. Previamente a la aplicación del instrumento de observación, fue sometido a una validación por jueces expertos. Su consistencia interna de acuerdo con el alfa de Cronbach fue de 0.78 para la escala total y por encima de 0.75 para cada subescala. El maestro calificó cada ítem entre 0 y 10 ( 0 = nunca, $10=$ casi siempre $)$.

b) The BarOn Emotional Quotient Inventory: Youth Version (EQ-i:YV); versión en español (BarOn-ICE:NA), reducida y adaptada por Ugarriza y Pajares (2004)

Para evaluar el efecto del programa en niños de ciclo medio y superior de educación primaria (9-12 años) se aplicó este instrumento, que se ha utilizado previamente con este rango de edad por otros autores, y se había demostrado su fiabilidad y validez (Sotil, et al, 2005; Ugarriza y Pajares, 2005). La versión corta del inventario consta de 30 ítems y establece un cociente emocional total y uno para cada una de las siguientes escalas: interpersonal, intrapersonal, adaptabilidad y manejo del estrés (Bar-On y Parker, 2000). La escala interpersonal evalúa el grado en que las relaciones sociales son satisfactorias, e incluye habilidades como la empatía y la escucha, y comprender y apreciar los sentimientos de los demás. La escala intrapersonal mide la capacidad para entender las emociones propias así como la capacidad de expresar los sentimientos y necesidades. La escala de adaptabilidad 
evalúa el grado de flexibilidad y realismo al hacer frente a los cambios y resolver problemas. La escala de manejo del estrés mide la capacidad de regular la impulsividad, a mantener la calma y trabajar bien bajo presión.

\section{Análisis de Datos}

Una vez más, debido a la distribución normal de los errores y de la relación lineal entre las variables dependientes e independientes, los datos se sometieron a un análisis de modelo lineal general.

\section{Resultados del Estudio 2}

\section{a) Los niños de 6-8 años}

La normalidad de la muestra se determinó mediante la prueba de KolmogorovSmirnov, que se aplicó a la puntuación global de la escala y a las puntuaciones en las cinco dimensiones ( $p>0,05$ en todos los casos). A partir de los resultados se aplicó el modelo lineal general. La Tabla 3 muestra los estadísticos descriptivos obtenidos para cada una de las cinco dimensiones y para el desarrollo emocional global.

\section{Resultados del programa}

La aplicación del modelo lineal general con 1 grado de libertad para las variables competencias emocionales obtuvieron los siguientes resultados:

La conciencia emocional. Hubo un aumento significativo $(F=58,799, \mathrm{p}<.01)$ en las puntuaciones después de la intervención (etapa post-test) tanto para el GE como el GC. Existe también un efecto significativo entre los grupos $(F=36,27, \mathrm{p}<01)$. El principal hallazgo fue la interacción significativa entre grupo y fase, que muestra que sólo el GE mejoró significativamente después de la intervención $(\mathrm{F}=80.00, \mathrm{p}<0.01)$.

Regulación emocional. Experimenta un aumento significativo posterior a la intervención $(\mathrm{F}=5,20, \mathrm{p}<.05)$ tanto para el GE como para el GC, aunque el nivel de 
significación fue mayor $(\mathrm{F}=15.12, \mathrm{p}<.01)$ en el grupo de intervención. Sin embargo, el resultado principal fue la interacción significativa ( $\mathrm{f}=14,97, \mathrm{p}<.01$ ) entre grupo y fase que demuestra que sólo el grupo de intervención incrementa significativamente la regulación emocional después del programa.

Autonomía emocional. Hubo un aumento significativo después de la intervención (f = $16,32, \mathrm{p}<.01)$ tanto para el GE como para el GC, así como un efecto significativo entre los dos grupos $(\mathrm{f}=.24, \mathrm{p}<.01)$ a favor del GE. El resultado principal fue la interacción significativa entre grupo y fase que demuestra que sólo el GE mejoró significativamente después de la intervención $(\mathrm{F}=17,67, \mathrm{p}<0,01)$.

La competencia social. La competencia social aumentó significativamente después de la intervención ( $\mathrm{f}=16,26, \mathrm{p}<.01$ ), y también hubo un efecto significativo entre grupos $(\mathrm{F}=$ $4,81, p=0,03)$. Una vez más, el principal resultado fue la interacción significativa entre grupo y fase que demuestra que sólo el GE incrementa significativamente en competencia social después del programa $(\mathrm{f}=27,97, \mathrm{p}<0,01)$.

Competencias para la vida y el bienestar. Estos aspectos también experimentaron una mejora significativa después de la intervención ( $\mathrm{f}=4,09, \mathrm{p}<0,05$ ). No hubo diferencias significativas entre grupos $(\mathrm{F}=0,57, \mathrm{p}=.45)$. Sin embargo, el hallazgo principal fue la interacción significativa entre grupo y fase que demuestra que sólo el GE mejora significativamente en competencias para la vida y el bienestar después de la intervención $(\mathrm{F}=$ $4,83, \mathrm{p}=0,03)$.

Competencia emocional general. Mejora significativamente ( $f=11,59, p<0,01$ ) después de la intervención. No hubo diferencias entre grupos $(F=2,94, p=.93)$. El principal resultado fue la interacción significativa entre grupo y fase que demuestra que solo el grupo de intervención incrementa los valores generales $(\mathrm{F}=21,20, \mathrm{p}<.01)$ tal como se puede ver en la Figura 1. 


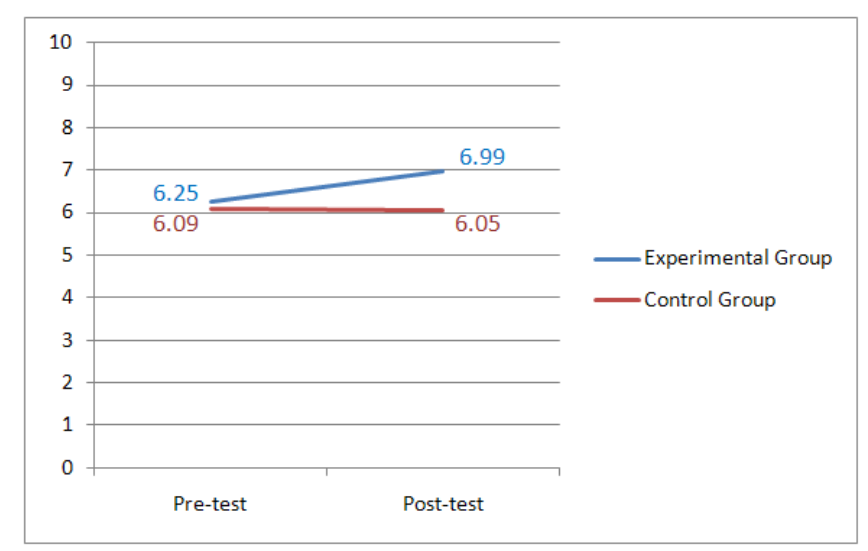

Figura 1. Cambio en el total de la escala después de la intervención

b) Los niños de 9-12 años

En la Tabla 3 se presentan los resultados descriptivos de los seis factores del test Bar-On.

Table 3. Estadísticos descriptivos de la escala aplicada a niños de 6-8 años

\begin{tabular}{llcccc}
\hline & & Pre-test & \multicolumn{3}{c}{ Post-test } \\
\hline \multirow{2}{*}{ EG } & & Mean & SD & Mean & SD \\
Conciencia emocional & 5.75 & $(.79)$ & 7.48 & $(.32)$ \\
& Regulación emocional & 6.47 & $(1.39)$ & 7.38 & $(1.04)$ \\
& Autonomía emocional & 6.25 & $(1.12)$ & 7.04 & $(.35)$ \\
& Competencia social & 6.57 & $(.937)$ & 7.71 & $(.93)$ \\
& Competencias de vida y bienestar & 6.17 & $(1.17)$ & 6.90 & $(1.29)$ \\
& Total & 6.25 & $(.87)$ & 6.99 & $(69)$ \\
\hline \multirow{2}{*}{ CG } & Conciencia emocional & 5.26 & $(.82)$ & 5.47 & $(.78)$ \\
& Regulación emocional & 5.96 & $(.93)$ & 5.7 & $(1.03)$ \\
& Autonomía emocional & 6.71 & $(.97)$ & 6.71 & $(.91)$ \\
& Competencia social & 6.53 & $(1.28)$ & 6.3 & $(1.40)$ \\
& Competencias de vida y bienestar & 6.75 & $(1.36)$ & 6.68 & $(1.38)$ \\
& Total & 6.09 & $(.83)$ & 6.05 & $(.87)$ \\
\hline
\end{tabular}

El análisis de acuerdo con el modelo lineal general de medidas repetidas en los resultados obtenidos para el factor intrapersonal del EQ-i: YV (versión en español) no mostró 
diferencias significativas entre fases ( $f=1,05, \mathrm{p}>.05)$, ni entre grupos $(\mathrm{F}=1,73, \mathrm{p}>0,05)$. Por el contrario, se observaron diferencias significativas entre grupos de edad $(F=6,57, p$ $<0,05)$. Los resultados también revelaron una interacción significativa entre fase y grupo* $(\mathrm{f}=$ 9,94, $\mathrm{p}<.01)$, mientras las puntuaciones del GC descienden las del GE aumentan.

El factor interpersonal mostró un aumento significativo $(F=33,81, p<0,01)$ después de la intervención. También había un efecto significativo entre grupos ( $\mathrm{f}=.03, \mathrm{p}<.01$ ), y en la edad $(\mathrm{F}=10,97, \mathrm{p}<0,05)$. Los resultados revelaron una interacción significativa entre fase y grupo * ( $\mathrm{f}=3,09, \mathrm{p}<.01)$, de tal manera que las puntuaciones del GC disminuían, mientras que las del GE aumentaban. Por último, también se observaron mejoras significativas en los resultados de los alumnos de 9-12 años del GE ( $f=1,21, \mathrm{p}<0,05)$.

En adaptabilidad se observó una mejora significativa ( $\mathrm{f}=119,25, \mathrm{p}<0,01$ ) después de la intervención. Sin embargo, no hubo ningún efecto significativo entre grupos. Se observó una interacción significativa $(\mathrm{f}=6,756, \mathrm{p}<0.01)$ entre fase y grupo*, de manera que las mientras las puntuaciones en el GC disminuían, las del GE aumentaban. También hubo una interacción entre grupo y * edad $(\mathrm{F}=0.58, \mathrm{p}=.02)$, a favor del alumnado de 9-12 años de edad en el GE.

El resultado principal en materia de gestión del estrés fue la interacción significativa entre Grupo y Fase que demuestra que sólo el grupo de intervención disminuye las sensación de estrés de forma significativa $(\mathrm{F}=0.10, \mathrm{p}<.01)$ partiendo de una situación inicial en la que no existían diferencias significativas en la fase $(\mathrm{f}=3,59, \mathrm{p}=0,08)$ y tampoco entre grupos ( $\mathrm{f}$ $=69, \mathrm{p}=.72$ ).

En inteligencia emocional, se observó una mejora significativa $(\mathrm{F}=1,09, \mathrm{p}<0,01)$ después de la intervención, y también hubo un aumento significativo en relación a la edad ( $\mathrm{F}$ $=6,56, \mathrm{p}=0,03)$. El resultado principal fue la interacción significativa entre fase y grupo * (f $=25,52, \mathrm{p}<.01)$, es decir, mientras las puntuaciones del GC descendían, las del GE aumentaban. 


\section{Discusión del Estudio 2}

Los datos obtenidos, tanto con la escala de observación, que se utilizó para evaluar el desarrollo emocional del alumnado de 6-8 años, como con el test de Bar-On, que se administró al alumnado de entre 9-12 años, permiten afirmar que las mejoras observadas son debidas a la intervención realizada. En los alumnos más pequeños se ha alcanzado una mejora significativa en todas las dimensiones de la competencia emocional trabajadas con el programa. Por su parte el contraste entre fase inicial y final mediante el test de Bar-On permite afirmar que los niños de ciclo medio y superior han experimentado una mejora en cuatro componentes: intrapersonal, interpersonal, adaptabilidad e inteligencia emocional. Sin embargo, en gestión del estrés no experimentaron mejoras significativas, aunque hay que señalar que este aspecto no fue abordado mediante el programa de educación emocional desarrollado. Como propuesta de futuro se podría incluir alguna actividad relacionada con la dimensión competencias para la vida a fin de trabajar este aspecto.

Los resultados del estudio proporcionan una nueva evidencia para apoyar la idea de que las competencias emocionales pueden desarrollarse a través de programas de educación emocional. En consecuencia, los sistemas educativos deberían considerar seriamente la incorporación de programas destinados al desarrollo de estas competencias, que en definitiva favorecen el bienestar personal y social del alumnado.

\section{Discusión General}

El propósito de los dos estudios presentados en este artículo era evaluar el impacto de un programa de entrenamiento de la competencia emocional utilizado, inicialmente, con los maestros, y posteriormente con los alumnos. El programa se basó en el modelo de competencia emocional de Bisquerra y Pérez (2007), que considera la educación emocional como un proceso educativo continuo y permanente que fortalece el desarrollo de competencias emocionales, consideradas como un elemento esencial del desarrollo humano. El objetivo de tal proceso era capacitar a la persona para la vida y, por lo tanto, incrementar su bienestar personal y el de su sociedad (Bisquerra, 2000). El programa implementado estaba concentrado en 30 horas de formación para los maestros y cerca de 20 horas de formación para los escolares. El objetivo era desarrollar en maestros y alumnos la conciencia emocional, 
regulación emocional, autonomía emocional, competencia social, y competencias para la vida y el bienestar. Ambos estudios mostraron una mejora en las competencias emocionales de los participantes, lo que demuestra que el programa es eficaz. Los maestros en el grupo experimental mejoraron sus competencias emocionales, especialmente en cuanto a sus habilidades de regulación emocional y competencias sociales. También redujeron sus niveles de estrés. Además, el programa dio lugar a una mejora en el clima institucional de la escuela. Por otra parte, cuando los maestros aplican el programa de educación emocional con sus alumnos, este último también mostró una mejoría en las competencias emocionales.

Aunque, en los últimos años, otros programas diseñados para desarrollar las competencias emocionales han demostrado ser eficaces, Nelis et al. (2009) señalan que la mayoría de estos estudios carecen de una base teórica sólida y no utilizan un grupo control para evaluar la efectividad del programa. El programa descrito aquí es el primero que se basa en el modelo de la competencia emocional de Bisquerra y Pérez, que incorpora las competencias cuya importancia ha sido demostrada por otros investigadores, pero que otros modelos de la IE no incluyen, por ejemplo, la autonomía emocional (Noom, 2001), aprender a fluir (Csikszentmihalyi, 1990) y competencias para la vida (Hopson et al., 1987). También hay que señalar, como lo destaca Nelis et al. (2009), que hay pocos estudios anteriores de programas de educación emocional que hayan utilizado grupos control. Mediante el uso de un grupo experimental y un grupo control este estudio fue capaz de comparar los efectos del programa.

Catalano et al. (2002) demuestran que los programas de educación emocional más exitosos comparten tres características: trabajan en el desarrollo de habilidades, se centran no sólo en los propios niños sino también en su entorno, y que implican una intervención que dura por lo menos nueve meses. El presente estudio comparte todas estas características. En concreto, la formación hace hincapié en la construcción de las cinco competencias emocionales incluidas en el modelo de Bisquerra y Pérez (2007), y utiliza una serie de actividades experienciales, juegos de rol y dinámicas de grupo. Una de las maneras en que se puede mejorar el clima institucional es aumentando la competencia emocional de los maestros para reducir el burnout (Brackett, et al, 2010). En el presente estudio este hecho se refleja en los niveles más bajos de estrés reportados por los maestros, y la mejora concomitante en el clima institucional. Un último punto a destacar es que la formación que se ofreció a los 
maestros y alumnos tomó la forma de una intervención a largo plazo (nueve meses en ambos casos).

Al igual que cualquier investigación evaluativa, el presente estudio tiene ciertas limitaciones. En primer lugar, algunos de los instrumentos de medición se han diseñado específicamente para este estudio. Aunque la mayoría de ellos mostró una buena fiabilidad y fueron validados por jueces expertos, la escala de estrés tenía una fiabilidad relativamente baja, lo que pone en peligro los resultados obtenidos con esta medida. Sin embargo, esta medida se correlacionó en la dirección esperada con la medida del clima institucional, apoyando así su validez. Otra limitación fue que los datos fueron recolectados a través de medidas de auto-informe o por medio de observaciones de los maestros, produciendo un tipo de información subjetiva que es propenso a un cierto grado de sesgo. Sin embargo, hay dos problemas con el uso de medidas directas de capacidades emocionales reales: en primer lugar, no existen medidas de capacidad para los niños en el rango de edad investigados aquí, y en segundo lugar, la capacidad de las medidas directas son costosos y requieren mucho tiempo para administrarlas (Humphrey et al., 2010). Otra limitación a tener en cuenta es que se trabajó con una muestra de conveniencia que puede no ser completamente representativa. Por consiguiente, sería aconsejable repetir el estudio con una muestra aleatoria de mayor tamaño con el fin de permitir una mayor generalización de los resultados. Un diseño de investigación longitudinal también sería necesario para investigar el grado con el que los resultados se mantienen a largo plazo.

Se requiere una futura investigación para examinar si la eficacia de este programa también podría extenderse a los importantes resultados del desarrollo, tales como la mejora del rendimiento escolar de los niños, reduciendo su comportamiento agresivo y niveles de conflicto en la escuela, y el aumento de su bienestar general. También sería importante investigar si un programa basado en el mismo modelo y principios podría aplicarse con resultados similares en alumnos de secundaria. Otro objetivo importante para una investigación futura sería complementar la formación de los programas que se ofrece a los maestros y alumnos con la de los padres.

A pesar de las limitaciones mencionadas anteriormente este estudio confirma que las competencias emocionales pueden ser mejoradas a través de una intervención sistemática y bien estructurada que se basa en un modelo teórico sólido, que es capaz de desarrollar habilidades emocionales y que se dirige no sólo a los alumnos sino también al entorno escolar. En términos de la evaluación de programas de educación emocional hay que señalar 
que este estudio utiliza un grupo control, que no es el caso de la mayoría de investigaciones anteriores de este tipo. También ha desarrollado una nueva medida de observación de las competencias emocionales de los niños de corta edad, una medida que puede ser útil para futuros estudios ya que los niños pequeños se sabe que tienen dificultades para responder los cuestionarios de auto-informe.

Aunque el siglo XX trajo muchos avances tecnológicos, también ha ido acompañado de un cierto deterioro en la capacidad de gestionar nuestras emociones, y esto se puede ver a través de muchas formas: conflictos, violencia, ansiedad, estrés, depresión, etc (Bisquerra, 2000). En el contexto escolar a menudo se observa situaciones de analfabetismo emocional, tales como: la falta de control emocional, conducta impulsiva, bajo rendimiento debido al estrés, el acoso y el maestro burnout (Crary, 1998). En este sentido, diversos estudios han relacionado la intimidación y el desgaste con sentimientos de ira, baja autoestima, soledad y otras dificultades emocionales, tanto en los agresores como en sus víctimas (Mearns \& Cain, 2003). Esto pone de relieve la necesidad de actuar de forma preventiva en los primeros años de la educación, así como en el contexto de la formación del profesorado. Pérez-González (2008) señala que la educación emocional de los niños es una tarea de las escuelas, las familias y las comunidades. Este estudio presenta un programa de educación educativo para las escuelas el cual tiene éxito en mejorar las habilidades emocionales de los niños.

\section{Agradecimientos}

Esta investigación ha sido realizada gracias a la financiación del Ministerio de Ciencia e Innovación de España, Dirección general de programas y transferencia de conocimiento (SEJ2007-65505/EDUC) y a la ayuda ARCE (Agrupación de Investigación en Ciencias de la Educación) de la Facultad de Pedagogía de la Universidad de Barcelona. 


\section{Referencias}

Bar-On, R., \& Parker, J. D. A. (2000). The Bar-On Emotional Quotient Inventory: Youth Version (EQ-i:YV) Technical Manual. Toronto, Canada: Multi-Health Systems.

Bechara, A., Tranel, D., \& Damasio, A. R. (2000). Poor judgement in spite of high intellect: Neurological evidence for emotional intelligence. In R. Bar-On \& J. D. A. Parker (Eds.), The Handbook of Emotional Intelligence: Theory, Development, Assessment, and Application at Home, School, and in the Workplace (pp. 192-214). San Francisco: Jossey-Bass.

Benítez, J. L., Fernández, M., Justicia, F., Fernández E., \& Justicia A. (2011). Results of the Aprender a Convivir Program for development of social competence and prevention of antisocial behavior in four-year-old children. School Psychology International, 32, 3 19.

Bisquerra, R. (2000). Educación emocional y bienestar. Barcelona: Praxis.

Bisquerra, R., \& Pérez-Escoda, N. (2007). Las competencias emocionales. Educación XXI, 10, 61-82.

Bisquerra, R. (2009) Psicopedagogía de las emociones. Madrid: Síntesis.

Brackett, M. A., Palomera, R., Mojsa-Kaja, J., Reyes, M. R., \& Salovey, P. (2010), Emotionregulation ability, burnout, and job satisfaction among British secondary-school teachers. Psychology in the Schools, 47, 406-417

Catalano, R. F., Berglund, M. L., Ryan, Jeanne A. M., Lonczak, Heather S., \& Hawkins, J. D., (2002). Positive youth development in the United States: Research findings on evaluations of positive youth development programs. Prevention \& Treatment, 5, 15223736.

Cherniss, C. (2000). Social and emotional competence in the workplace. In R. Bar-On \& J. D. A. Parker (Eds.), The Handbook of Emotional Intelligence: Theory, Development, Assessment, and Application at Home, School, and in the Workplace (pp. 433-459). San Francisco: Jossey-Bass.

Clouder C., Dahlin B., Diekstra R., Berrocal P. F., Heys B., Lantieri L., Paschen H., et al., (2008) Social and Emotional Education: An International Analysis. Santander: Fundación Marcelino Botín. Retrieved July, 72010 from http://educacion.fundacionmbotin.org/ficheros_descarga/pdf/EN/preface.pdf 
Collaborative for Academic, Social, and Emotional Learning (CASEL) (2003). Safe and sound: An educational leader's guide to evidence-based social and emotional learning (SEL) programs. Chicago: Author.

Csikszentmihalyi, M. (1990). Flow: The Psychology of Optimal Experience, New York: Harper \& Row.

Crary, E. (1994). Crecer sin peleas: Cómo enseñar a los niños a resolver conflictos con inteligencia emocional. Barcelona: Integral.

Di Fabio, A., \& Kenny, M. E. (2011). Promoting emotional intelligence and career decision making among Italian high school students. Journal of Career Assessment, 19, 21-34.

Extremera, Fernández-Berrocal \& Durán, (2003) Inteligencia emocional y burnout en profesores. Encuentros en Psicología Social, 1, 260-265.

Extremera, N. \& Fernández-Berrocal, P. (2004). El papel de la inteligencia emocional en el alumnado: evidencias empíricas. Revista Electrónica de Investigación Educativa, 6(2), 117.

Fernández-Berrocal P., \& Ruiz Aranda, D. (2008). La inteligencia emocional en la educación. Electronic Journal of Research in Educational Psychology, 6, 421-436.

Gardner, H. (1993). Multiple intelligences: The theory in practice. New York: Basic Books.

Greenberg, M., Weissberg, R., O’Brien, U., Zins, J., Fredericks, L., Resnik, H., \& Elias, M. (2003). Enhancing School-Based Prevention and Youth Development Through Coordinated Social, Emotional, and Academic Learning. American Psychologist, 58 $(6 / 7), 466-474$.

Goleman, D. (1995). Emotional Intelligence. Why it can matter more than IQ. New York: Bantam Books. (Spanish version: Inteligencia emocional, Barcelona, Kairós, 1996; 15ª edición 1997).

Hedlund, J., \& Sternberg, R. J. (2000). Too many intelligences? Interpreting social, emotional, and practical intelligence. In R. Bar-On \& J. D. A. Parker (Eds.), The Handbook of Emotional Intelligence: Theory, Development, Assessment, and Application at Home, School, and in the Workplace, 136-167. San Francisco: Jossey-Bass.

Hopson, B. \& Scally, M. (1987). Lifeskills teaching programmes. No.2. Lifeskills Associates, Leeds, U.K

Humphrey, N., Kalambouka, A., Wigelsworth, M., Lendrum, A. Lennie, C., \& Farrell, P. (2010). New Beginnings: evaluation of a short social-emotional intervention for primary-aged children. Educational Psychology, 30 (5), 513-532. 
Mayer, J. D., Caruso, D. R., \& Salovey, P. (1999). Emotional intelligence meets traditional standards for an intelligence. Intelligence, 27 (4), 267-298.

Mayer, J. D., \& Salovey, P. (1993). The intelligence of emotional intelligence. Intelligence, 17 (4), 433-442.

Mearns, J., \& Cain, J. E. (2003). Relationships between teachers' occupational stress and their burnout and distress: Roles of coping and negative mood regulation expectancies. Anxiety, Stress and Coping, 16 (1), 71-82.

Nelis, N., Quoidbach, J., Mikolajczak, M., \& Hansenne, M. (2009). Increasing emotional intelligence: (How) is it possible? Personality and Individual Differences 47, 36-41.

Noom, M.J., Deković M., \& Meeus W. H. J. (2001). Conceptual Analysis and Measurement of Adolescent Autonomy. Journal of Youth and Adolescence, 5, 30, 577-595.

Pérez-Escoda, N., Bisquerra, R., Filella, G., \& Soldevila, A. (2010). Construcción del cuestionario de desarrollo emocional de adultos (QDE-A). Revista Española de Orientación y Psicopedagogía, 21, 367-379.

Pérez-González, J.C. (2008). Propuesta para la evaluación de programas de educación socioemocional. Electronic Journal of Research in Educational Psychology, 6, 523546. ISSN 1696-2095.

Petrides, K. V., Frederickson, N., \& Furnham, A. (2004). The role of trait emotional intelligence in academic performance and deviant behavior at school. Personality and Individual Differences, 36, 277-293.

Saarni, C. (2000). Emotional competence: A developmental perspective. In R. Bar-On \& J. D. A. Parker (Eds.), The Handbook of Emotional Intelligence: Theory, Development, Assessment, and Application at Home, School, and in the Workplace, (pp. 68-91). San Francisco: Jossey-Bass.

Salovey, P., Bedell, B., Detweiler, J. B., \& Mayer, J. D. (2000). Current directions in emotional intelligence research. In Lewis, M. \& Haviland-Jones, J. M. (Eds.) Handbook of emotions. New York: Guilford Press, 504-520.

Sotil, A., Escurra, L., Huerta, R., Rosas, M., Campos, E., \& Llaños, A. (2008). Efectos de un programa para desarrollar la inteligencia emocional en alumnos del sexto grado de educación primaria. Revista de Investigación en Psicología, 11(2), 55-65.

Sroufe, L. A. (2000). Early relationships and the development of children. Infant Mental

Health Journal, 21, 67-74. 
Triliva S., \& Poulou M. (2006). Greek Teachers' Understandings and Constructions of What Constitutes Social and Emotional Learning. School Psychology International, 27, 315338.

Ugarriza, N., \& Pajares, L. (2004). Adaptación y estandarización del Inventario de Inteligencia Emocional de BarOn ICE: NA, en niños y adolescentes. Manual técnico. Lima: Edición de las autoras.

Ugarriza, N., \& Pajares, L. (2005). La evaluación de la inteligencia emocional a través del inventario de BarOn ICE: NA, en una muestra de niños y adolescentes. Persona, 8, $11-58$. 